\title{
CONSIDERACIONES PARA UN TRATAMIENTO DOGMÁTICO DE LAS CATEGORÍAS DE AUTOEJECUTABILIDAD Y NO-AUTOEJECUTABILIDAD DE LAS NORMAS DE LOS TRATADOS INTERNACIONALES
}

\author{
CONSIDERATIONS FOR TREATMENT
}

OF DOGMATIC SELF-EXECUTING AND NON-SELF-EXECUTING

CATEGORIES STANDARDS OF INTERNATIONAL TREATIES

QUELQUES CONSIDÉRATIONS POUR

UNE ANALYSE DOGMATIQUE DE CATÉGORIES D’AUTOEXÉCUTION

ET NON AUTOEXÉCUTION DES RĖGLES DES TRAITÉS INTERNATIONAUX

George Lambeth Vicent ${ }^{* *}$

\begin{abstract}
RESUMEN
El propósito de este trabajo es proporcionar elementos dogmáticos necesarios para conceptualizar operativamente las categorias de autoejecutabilidad y no autoejecutabilidad de las normas contenidas en los tratados internacionales. Con una referencia crítica a la discusión doctrinaria y a la jurisprudencia de los Estados Unidos, se busca clarificar algunos problemas dogmáticos que están presentes en el Derecho Constitucional chileno. Como se intentará mostrar, el tratamiento que la doctrina nacional hace de estas categorias, sobre todo desde la incorporación en la jurisprudencia del Tribunal Constitucional (fallo sobre el Convenio 169 OIT), opera en niveles superficiales, impidiendo establecer de manera clara cuáles son los elementos dogmáticos y positivos que están en conflicto y cómo darles una solución coherente y sistemática.

Palabras Clave: Derecho Internacional - Derecho Constitucional - Tratado Internacional Autoejecutabilidad - No Autoejecutabilidad

ABSTRACT

The purpose of this paper is to provide dogmatic elements required to operatively conceptualize the categories of self-executing and non-self-executing rules and standards contained in international treaties. With a critical reference to the doctrinal discussion and case law of the United States, it seeks to clarify some dogmatic issues at present in the Chilean constitutional law. As will be explained, the treatment that the national law makes of these categories, especially since the incorporation in the jurisprudence of the Constitutional Court (ruling on ILO Convention 169), operates in surface levels, preventing to establish clearly which dogmatic and positive elements are conflictive, and how to give a coherent and systematic solution.
\end{abstract}

\footnotetext{
* Artículo recibido el 30 de abril de 2015 y aceptado para su publicación el 16 de junio de 2015.

${ }^{* *}$ Abogado. Licenciado en Ciencias Jurídicas y Sociales de la Universidad de Chile. Magíster (C) en Derecho Público por la Universidad de Chile. Profesor Departamento Derecho Público Universidad Alberto Hurtado. Abogado Asesor Coordinación Legislativa Ministerio de Hacienda, Gobierno de Chile. Correspondencia a: Correo electrónico george.lambeth@gmail.com.
} 
KEYWORDS: Constitutional Law - International Law - International Treaty - Self-executing - Not self-executing

RESUMÉ

Cette chronique envisage de donner élements dogmatiques nécessaires pour arriver à une définition pratique des categories d'auto-exécution et non auto-exécution des règles des traités internationaux. Dans une optique critique au débat doctrinal et à la jurisprudence des Etats Unis, on a pour but de éclaircir quelques problèmes dogmatiques au sein du droit constitutionnel chilien. On envisage de démontrer que l'analyse que la doctrine nationale fait de ces catégories, surtout depuis l'incorporation dans la jurisprudence du Tribunal Constitutionnel (arrêt sur le Convetion 169 de l'OIT), a lieu dans des niveaux superficiaux, ce qui empêche d'établir d'une manière claire quels sont les élements dogmatiques et positifs qui sont en conflit et comment leur donner une solution cohérent et systématique.

MoTS CLÉS: Droit international - Droit constitutionnel - Traité international - Auto exécution - Non auto-exécution

\section{Consideraciones PRELIMINARES.}

EL ORIGEN DE LAS CATEGORÍAS EN EL DERECHO DE LOS ESTADOS UNIDOS

En sentencia rol No 309 de 2000 sobre el Convenio 169 de la OIT, el Tribunal Constitucional (en adelante "TC") incorporó expresamente las categorías de autoejecutabilidad y no autoejecutabilidad ${ }^{1}$ (o, en su terminología originaria, el carácter self-executing y non self-executing, utilizándose indistintamente en lo que sigue) de los tratados internacionales y las normas contenidas ${ }^{2}$.

Los presupuestos de su incorporación fueron los siguientes: "Los tratados, para su aplicación en el orden interno de un pais, pueden contener dos tipos de cláusulas, denominadas por la doctrina 'self-executing' $y$ 'non self-executing'. Las primeras, son las que tienen el contenido y precisión necesarias que las habilita para ser aplicadas sin otro trámite como fuente del derecho interno (...). Las segundas, son aquellas que requieren para su entrada en vigencia de la dictación de leyes, reglamentos o decretos que las implementen y, en tal evento, las haga aplicables como fuente del derecho interno" 3 .

La incorporación de estas categorías en la jurisprudencia del TC plantea una serie de preguntas al Derecho Constitucional. En un primer momento, estas categorías suponen responder la pregunta de la aplicabilidad de los Tratados Internacionales en el Derecho chileno. Pero la incorporación supuso un elemento adicional de consideración. En efecto, ¿`cuáles son aquellas normas contenidas en

\footnotetext{
${ }^{1}$ Sin perjuicio de que la doctrina y cierta jurisprudencia ya había utilizado las categorías con anterioridad, es esta sentencia del TC la que inicia el debate proveyendo criterios de sistematización. Por lo mismo, sin desconocer menciones anteriores, este trabajo asume como punto de partida la STC rol No 309, 4 de agosto de 2000

2 Sin perjuicio que la referencia correcta es sobre las disposiciones normativas del tratado internacional y no sobre sus normas, según se verá, siguiendo el sentido que le otorga a esta distinción, VON WRIGHT, George Henrik (1963). Norm and Action. A Logical Enquiry. London: Routledge \& Kegan Paul, pp. 93 y ss.

${ }^{3}$ STC rol No 309.
} 
los Tratados Internacionales que requieren para su entrada en vigencia, no solo la satisfacción del procedimiento de incorporación de los tratados internacionales al derecho interno, sino la dictación de leyes o reglamentos que las habiliten para poder ser consideradas por los tribunales como razones institucionales en la resolución de los conflictos sometidos a su conocimiento? ¿Cuál es el criterio de distinción entre los tratados o sus normas que son autoejecutables y los que no?

El propósito de estas notas será intentar proveer algunos criterios para el tratamiento dogmático de estas preguntas y para conceptualizar operativamente las categorías de autoejecutabilidad y no autoejecutabilidad de las disposiciones contenidas en los Tratados Internacionales, bajo el supuesto que su incorporación definitiva en nuestro medio es irreversible.

Parte central de la conclusión de este ejercicio es vincular el sentido correcto de estas categorías con un origen histórico e institucional especial en la tradición jurídica estadounidense. Lo que se pretende explicar es el surgimiento y función de estas categorías en el Derecho norteamericano para después ver si acaso esa discusión nos puede clarificar la adopción en nuestra jurisprudencia y doctrina de estas categorías. Las categorías tienen su origen en condiciones históricas y políticas muy especiales, que dicen relación con problemas jurídicos propios del derecho norteamericano que no son reproducible vis a vis al sistema chileno. Esto quiere decir que la adopción de estas categorías, sin considerar esta especial condición, solo trae aparejada una serie de problemas metodológicos relevantes que, como se verá, han contribuido a una discusión que no ha logrado proveer los criterios de aplicación de estas categorías.

Sin perjuicio de lo anterior, varios de los supuestos que están detrás de la discusión norteamericana sí pueden ser útiles para la discusión nacional. Lo anterior porque lo que la doctrina comparada evidencia es, finalmente, un problema que poseen la mayoría de los sistemas jurídicos occidentales: la tensión entre su proyección internacional y la legitimidad del derecho interno.

Como señalan ZWEIGERT y KÖTZ ${ }^{4}$, el Derecho comparado asume como principio metodológico básico el de la funcionalidad (solo es posible la comparación de lo que cumple la misma función) y que este principio proviene de la constatación que los sistemas legales de todas las sociedades y tradiciones presentan, en general, los mismos problemas respecto de los cuales hay que encontrar una solución, una adopción errónea o superficial de soluciones o instituciones jurídicas comparadas que desconozca precisamente la verdadera función que cumple en el sistema jurídico comparado puede terminar siendo un problema mayor que el que se quiere solucionar.

En lo que sigue (2) se tratará muy esquemáticamente el origen de las categorías en el Derecho estadounidense y una referencia general al estado de la discusión

${ }^{4}$ Zweigert, Konrad and Kötz, Hein (1996). Introduction to Comparative Law. Oxford: Clarendon Press, Third Edition, pp. 34-35 y 40. 
doctrinaria. Un intento serio de operativizar estas categorías en el Derecho chileno tiene que considerar los elementos de la doctrina estadounidense. A continuación (3) presentará el problema de la autoejecutabilidad en el Derecho chileno. Los presupuestos de la discusión a nivel nacional nos permitirán establecer como diagnóstico (4) la poca profundidad con que estas categorías han sido tratadas pero, sobre todo, un equivocado tratamiento dogmático debido a un déficit doctrinario a nivel de Derecho comparado.

\section{REFLEXIONES A PROPÓSITO DEL ORIGEN}

DE LAS CATEGORÍAS. LOS SUPUESTOS INSTITUCIONALES TRAS SU APLICACIÓN

Hay consenso en que el origen de las categorías encuentra su momento inicial en la sentencia de Foster v. Neilson ${ }^{5}$, en 1829. Sin embargo, el conflicto que estas categorías suponen solucionar ha sido tratado mucho antes, especialmente en el caso de Ware v. Hylton ${ }^{6}$ (1796), donde lo que se discutía era precisamente si los tratados internacionales eran considerados una ley federal vinculante para todos los estados, en los términos que la Constitución de los EE.UU. lo declaraba, estando por sobre las leyes de los estados de la Unión o si en cambio habría algún presupuesto de aplicación adicional ${ }^{7}$.

A la decisión sobre Foster v. Neilson le siguió la sentencia sobre U.S. v. Percheman, de 1832, que resolvió justamente sobre el mismo Tratado Internacional que fuera motivo de disputa en Foster ${ }^{8}$. De ahí hasta el año 2008 (más de 200 años) en que se fallara en el caso Medellín v. Texas ${ }^{9}$, que no había sido tratado el asunto por la Corte Suprema. Las cortes distritales y estatales junto con la doctrina venían debatiendo profusamente sobre las implicancias de estas categorías en términos muy especiales, propios de la tradición jurídica de los EE.UU.

El debate puede graficarse en torno a dos posiciones bien marcadas. Por una parte, el enfoque que podríamos denominar internacionalista, que sostiene que la interpretación correcta de la Cláusula Suprema en conjunto con los fallos de la Corte Suprema suponen entender que habría una presunción por el carácter selfexecuting de los tratados internacionales. En tal sentido, los tratados internacionales serían directamente aplicables por las cortes y tribunales del país del mismo modo que lo sería una ley común y corriente o, incluso la propia Constitución (al menos en el sentido que lo define en Marbury v. Madison).

\footnotetext{
5 U.S. Supreme Court, Foster \& Elam v. Neilson, 27 U.S. 2 Pet. 253 (1829).

6 Ware v. Hylton, 3 U.S. 3 Dall. 199199 (1796).

${ }^{7}$ Acá el caso tenía que ver por un conflicto entre una ley del Estado de Virginia y un Tratado de Paz con el Reino Unido sobre las garantías a los créditos británicos en América.

8 United States v. Percheman, 32 U.S. 7 Pet. 51 (1832).

${ }^{9}$ Medellin v. Texas 552 U.S. 491 (2008).
} 
De otro lado, se encontraría los así denominados nacionalistas quienes afirman que una correcta interpretación de las fuentes lleva necesariamente a la conclusión de que habría una presunción de no autoejecutabilidad, que esa interpretación es respetuosa de la Cláusula Suprema y el rule of law y que las interpretación del Juez Marshall en Foster y Percheman son correctas sí, y solo sí, son leídas al modo que lo hace la Corte Suprema en el caso Medellín v. Texas.

El debate tiene el elemento en común, donde lo que se discute es si los tratados internacionales son aplicables de acuerdo a la Cláusula Suprema contenida en el artículo VI de la Constitución de los EE.UU. Esta Cláusula es lo que se ha denominado el germen de la doctrina que permea todo el Derecho Constitucional norteamericano que afirma que la Constitución es la ley suprema de la nación, con la característica de anular cualquier ley que sea contraria y respecto de la cual, los tribunales deben imponerla ${ }^{10}$.

El texto de la Cláusula Suprema establece que ${ }^{11}$ : "All Debts contracted and Engagements entered into, before the Adoption of this Constitution, shall be as valid against the United States under this Constitution, as under the Confederation. This Constitution, and the Laws of the United States which shall be made in Pursuance thereof; and all Treaties made, or which shall be made, under the Authority of the United States, shall be the supreme Law of the Land; and the Judges in every State shall be bound thereby, any Thing in the Constitution or Laws of any state to the Contrary notwithstanding".

Una lectura imparcial y literal sugiere que los Tratados Internacionales son ley suprema de la nación y que los jueces están vinculados en el mismo sentido que respecto a la Constitución y a las demás leyes. Como propone Vásquez ${ }^{12}$, una correcta lectura de esta Cláusula supone realizar una comparación con el estatus de los tratados internacionales en el Reino Unido. Desde la Gloriosa Revolución de 1688 que se considera que los tratados internacionales celebrados por la Corona que modifican el derecho doméstico del Reino Unido, deben ser aprobados posteriormente por el Parlamento. Un tratado internacional celebrado por la Corona, no tiene fuerza vinculante en el derecho interno a menos que el Parlamento lo apruebe en un acto legislativo especial y posterior. Una interpretación correcta de la Cláusula Suprema, añade Vásquez, supone un giro de esta regulación, en la medida que establece que para todos los estados de la Unión, un Tratado Internacional es vinculante en el mismo sentido que lo sería la Constitución y las leyes promulgadas a su efecto.

\footnotetext{
${ }^{10}$ Un relato particularmente interesante del desarrollo de esta concepción, desde una perspectiva histórica, puede verse en Morison, Samuel Eliot; Commager, Henry Steele y Leuchtenburg, William E. (2013). Breve Historia de los Estados Unidos de América. México D.F.: Fondo de Cultura Económica, p. 148.

${ }^{11}$ Se consultó directamente la versión de la Constitución en el sitio web del Congreso de los Estados Unidos, disponible en: <http://www.senate.gov/civics/constitution_item/constitution.htm> [Consulta: 19 enero 2015].

${ }^{12}$ VÁzquez, Carlos Manuel (2008). "Treaties as Law of the Land: The Supremacy Clause and the Judicial Enforcement of Treaties”. Harvard Law Review, Vol. 122, pp. 613-616.
} 
En esa interpretación, la Cláusula Suprema fue la solución a uno de los principales vicios que poseían los Artículos de la Confederación ${ }^{13}$. En efecto, la primera formulación de los Artículos de la Confederación señalaba que el Congreso tenía la potestad para celebrar los tratados Internacionales con las naciones extranjeras pero no establecía un mecanismo para que los estados de la confederación hicieran coactivamente aplicables dichos tratados por sus cortes ${ }^{14}$. Así, la formulación original de los Artículos de la Confederación, en un ánimo de unificación de cara al escenario internacional pero respetuoso de las autonomías federales, tenía el problema de diseño institucional de hacer vinculantes las obligaciones internacionales en los propios estados de la Confederación. Ese problema se manifestaba en que efectivamente no había un verdadero mecanismo de implementación de los tratados internacionales en las cortes y los tribunales de los estados.

Esta tensión no era más que una manifestación generalizada de la solución para que los estados implementaran las decisiones tomadas a nivel federal. Paradigmático es el caso de Tratado de Paz celebrado entre la nueva nación y el Reino Unido ${ }^{15}$. Entre los punto más importante de este tratado estaban las normas referidas a los créditos que poseían los acreedores británicos frente a los deudores norteamericanos. Dicho tratado establecía un reconocimiento de los créditos en conjunto con los mecanismos para su cobro. El problema radicaba en que los estados no reconocían las demandas para el cobro o dictaban leyes de prescripción de obligaciones, con lo que los acreedores británicos veían entrampados sus créditos. Esto naturalmente tensaba la relación internacional entre Washington y Londres.

Como señalan Sloss y VÁsQUez ${ }^{16}$, había acuerdo en la Convención Constitucional respecto a que la nueva Carta Suprema debería empoderar lo suficiente al Gobierno Federal para aplicar los tratados (y naturalmente fortalecer el propósito del Tratado de Paz). En otras palabras, el objetivo principal de la determinación de la Cláusula Suprema en lo que respecta a los tratados internacionales fue precisamente reconocer el estatus federal de éstos y la obligación de los estados de implementarlas.

El problema ha surgido justamente porque la denominada doctrina Foster estableció que existirían Tratados Internacionales que, sin perjuicio de la Cláusula Suprema, no serían self-executing ${ }^{17}$. En efecto, en Foster v. Neilson ${ }^{18}$ el Juez Marshall

13 Ídem.

${ }^{14}$ SLoss, David (2012). "Executing Foster v. Neilson: The Two-Step Approach to Analyzing Self-Executing Treaties”. Harvard International Law Journal, Vol. 53, pp. 309 y ss.

15 VÁzQuez (2008), p. 614.

16 VÁzquez (2008), pp. 613-616.

${ }^{17}$ La versión doctrinaria original de la denominada doctrina Foster, en VÁzQUeZ, Carlos Manuel (1995). "The four doctrines of self-executing treaties". The American Journal of International Law, Vol 89, No 4, pp. 695-723.

${ }^{18}$ Foster \& Elam v. Neilson, 27 U.S. 2 Pet. 253253 (1829). Al respecto, Bradley, Curtis (2009). “Self-execution and treaty duality". Supreme Court Review, pp. 131-182. También, Yoo, John (1999). "Treaties 
argumentó que "cuando los términos de una estipulación importan un contrato, cuando cualquiera de las partes se obliga a ejecutar un acto particular, el tratado se dirige al órgano político, no al judicial, y la legislatura debe ejecutar el tratado antes de que éste pueda devenir una regla para la Corte"19. La doctrina ha denominado a esta postura Foster-type non-self-execution ${ }^{20}$.

En términos generales entonces, un tratado o las normas contenidas en aquel son autoejecutables cuando puede ser aplicado directamente por los tribunales sin que requiera un acto legislativo adicional de implementación. En cambio, un tratado puede no ser autoejecutable cuando no puede aplicarse por los tribunales sin una intervención legislativa de implementación por parte del Congreso ${ }^{21}$.

La tensión inmanente en la discusión se encuentra determinada, en gran medida, por la consideración de los tratados internacionales como supreme law of the land de acuerdo a la Cláusula Suprema. Efectivamente, ¿cómo podría considerarse a un tratado internacional debidamente ratificado por el Congreso como supreme law of the land y no poder ser, al mismo tiempo, aplicado por las cortes y tribunales de los estados?

La pregunta que asalta a la doctrina es, finalmente, cómo conciliar, por un lado, el hecho de que hay tratados que no son autoejecutables en el sentido apuntado por la Corte Suprema estadounidense y, por otro lado, la consideración de la Cláusula Suprema de la Constitución de los EE.UU. Para hacer frente a esta tensión o anomalía que la doctrina Foster iniciara, Carlos VÁZQUEZ ha sostenido que "[t]he concept of a non-self-executing treaty is in tension with the Supremacy Clause's designation of treaties as 'law of the land', and that, as a result, our Constitution should be read to establish a presumption that treaties are self-executing" 22 . Esta es la que podría denominarse la formulación general de la postura internacionalista.

Frente a la presunción de la autoejecutabilidad defendida por los internacionalistas ha reaccionado parte importante de la doctrina ${ }^{23}$. Esta posición está explicitada en un autor como Yoo para quien "the original understanding of the constitutional

and Public Lawmaking: A Textual and Structural Defense of Non-Self-Execution”. Columbia Law Review, Vol. 99, pp. 2218-2258; Flaherty, Martin S. (1999). "History Right? Historical Scholarship, Original Understanding, and Treaties as 'Supreme Law of the Land'”. Columbia Law Review, Vol. 99, p. 2095.

${ }^{19}$ Foster \& Elam v. Neilson, 27 U.S. 2 Pet. 253253 (1829. Traducción libre.

${ }^{20}$ Curtis Bradley ha descrito esta posición de la siguiente manera: "A treaty may call for a governmental action, such as the appropriation of money or the creation of criminal liability that is thought to lie exclusively within the powers of the full Congress. Some treaty cases, like some constitutional and statutory cases, may be non-justiciable - for example, because of standing requirements or the political question doctrine. Or the case may depend upon the recognition of a private right of action, and the court may conclude that the treaty does not itself confer such a right of action. Finally, a court may conclude that a treaty was not intended to be judicially enforceable unless and until implemented by a political branch, usually Congress". BRADLEY (2009), p. 2.

21 VÁzQuez (1995), pássim.

${ }^{22}$ Cf. VÁzquez, Carlos Manuel (1999). "Laughing at Treaties”. Columbia Law Review, Vol. 99, pp. 2154-2217.

${ }^{23}$ Yoo (1999), pp. 2210 y ss. 
Founders was that treaties would not operate as domestic law when they (as is often the case today) addressed matters falling within the scope of Congress' legislative authority. As an alternative (...) there should at least be a presumption against treaty self-execution, such that the treaty makers would be required to issue a clear statement if they wanted a treaty to be self-executing" 24 .

La jurisprudencia estadounidense no fue clara en la provisión de criterios operativos para la adopción de una u otra postura, lo que ha fomentado el desarrollo de posturas marcadamente diferenciadas. No ha sido sino hasta el fallo de la Corte Suprema en Medellin v. Texas en $2008^{25}$ donde el panorama se ha ido clarificando en favor de una postura.

El fallo contenido en Medellín v. Texas ${ }^{26}$ modificó el panorama permitiendo un giro claro y más categórico para la presunción de la no autoejecutabilidad de los tratados internacionales. Aquí, la Corte Suprema sostuvo que "a treaty obligation of the United States to comply with a decision of the International Court of Justice (the international adjudicatory arm of the United Nations that sits in The Hague) was not self-executing and thus could not be applied by U.S. courts to override an otherwise valid state rule of criminal procedure. The Court also held that the President lacked the unilateral authority to compel state courts to comply with the International Court's decision" 27.

En este caso, la Corte rechazó lo que ella denominó “"[the] multifactor judgment by judgment' approach to self-execution, whereby courts would consider not only treaty text and drafting history, but also the treaty's subject matter". La Corte resolvió que dicho enfoque "would be too indeterminate and would give the courts too much discretion, thereby 'assign [ing] to the courts the political branches - the primary role in deciding when and how international agreements will be enforced"28.

Vistos estos antecedentes, podría sostenerse que tanto la doctrina como la jurisprudencia más reciente se han inclinado a favor de la tesis de la presuposición por la Foster-type non self-execution theory. En resumen, desde una descripción muy esquemática de las principales decisiones y comentarios de la doctrina, el debate

\footnotetext{
${ }^{24}$ Bradley (2009). Algunas cortes han aplicado directamente los tratados y han reconocido la presunción de autoejecutabilidad que señalaba Vázquez, por ejemplo en Kolovrat v Oregon, 366 US 187 (1961). Bradley enumera otros casos: Asakura v. City of Seattle, 265 US 332 (1924). Ware v. Hylton, 3 US 199 (1796). Otras cortes, sobre todo en fallos más contemporáneos, se han mostrado más reacias a reconocer esta presunción de autoejecutabilidad que permita "private judicial enforcement of treaties, especially multilateral treaties, so much so that it is arguable that the modern case law suggests a presumption against self-execution". Así, United States v. Emuegbunam, 268 F3d 377, 389 (6th Cir 2001). United States v. Li, 206 F3d 56, 60 (1st Cir 2000). Goldstar (Panama) S.A. v. United States, 967 F2d 965, 968 (5th Cir 1992).

25 Ídem.

${ }^{26}$ Medellin v. Texas, 552 U.S. (2008) (No 06-984).

27 Ídem.

${ }^{28}$ Bradley (2009). También, del mismo, Bradley, Curtis (2008). "Medellin: Intent, presumptions and non self-executing treaties". American Journal of International Law.
} 
sobre estas categorías es particularmente referido a la historia institucional norteamericana. Sin perjuicio de lo anterior, la discusión nos muestra que el núcleo de esto responde a un problema transversal en los sistemas jurídicos occidentales y contemporáneos, esto es, la tensión entre las obligaciones suscritas a nivel internacional, la nación como un actor en el escenario internacional, frente a los mecanismos de incorporación, como derecho interno, de esas obligaciones con todos los problemas de legitimidad que eso conlleva.

\section{Algunas consideraciones dogmáticas}

SOBRE EL DEBATE DE LA AUTOEJECUTABILIDAD DE LOS TRATADOS INTERnACiONALES EN EL Derecho CONSTITUCIONAL CHILENo

Como indica IWASAWA respecto de la discusión norteamericana ${ }^{29}$, es fundamental en el análisis del carácter autoejecutable de un tratado, la distinción entre la validez doméstica y la aplicabilidad. Esta distinción nos resulta familiar desde la teoría del derecho con la distinción entre validez (como criterio de pertenencia de una norma al sistema jurídico) y aplicabilidad en el caso concreto ${ }^{30}$. La aplicabilidad de una norma supone su validez de acuerdo a los criterios procedimentales de validez de cada sistema jurídico particular ${ }^{31}$. Así también, la aplicabilidad doméstica de los tratados internacionales o sus normas siempre suponen la validez del mismo. Como se verá, parte importante de los problemas que presenta el análisis de estas categorías en la doctrina nacional radican en desconocer esta distinción, especialmente considerando la doble naturaleza del tratado internacional, como norma de derecho interno y como norma de derecho internacional.

En tal sentido, resulta relevante aclarar los criterios procedimentales de validez del Derecho Constitucional chileno en lo que respecta a los tratados internacionales. $\mathrm{Al}$ respecto, las normas constitucionales referidas a la incorporación de tratados internacionales en el derecho interno son el artículo $32 \mathrm{No} 15$ y el artículo 54 No 1. Estas normas establecen de manera general las potestades para la celebración del tratado y la aprobación de este como norma de rango legal. En nuestro sistema, por lo tanto, todo el problema de la autoejecutabilidad surge una vez incorporado el tratado en el ordenamiento interno, en una etapa posterior a su ratificación. Luego,

\footnotetext{
${ }^{29}$ Ver al respecto el artículo seminal donde se establece esta cuestión. IWASAWA, Yuji (1986). "The doctrine of self-executing treatise in the United States: A critical analysis”. Virginia Journal of Internacional Law, No 26, pp. 627-692.

${ }^{30}$ La literatura es vasta. Cf. Moreso, Josep Joan (1997). La indeterminación del Derecho y la interpretación de la Constitución. Madrid: Centro de Estudios Constitucionales; Alchourrón, Carlos y Bulygin, Eugenio (1974). Introducción a la metodología de las ciencias sociales y jurídicas. Buenos Aires: Astrea. También, de los mismos, Alchourrón, Carlos y Bulygin, Eugenio (1991). Análisis lógico y derecho. Madrid: Centro de Estudios Constitucionales.

${ }^{31}$ Alchourrón y Bulygin (1974).
} 
la discusión no surge en la determinación, a priori, del carácter autoejecutable o no autoejecutable de un tratado internacional.

Pero el problema en el caso chileno es precisamente el contrario. Precisamente porque en el contexto nacional el debate ha desconocido la distinción entre la validez y la aplicabilidad del tratado internacional, el uso de estas categorías ha sido considerado como algo que puede ser resuelto antes de la incorporación del tratado al derecho interno porque el carácter autoejecutable viene determinado en el tratado mismo. El debate nacional podría dividirse en dos posiciones; de una parte están los autores que ven en la redacción normativa del tratado y sus disposiciones el contexto suficiente para determinar el carácter autoejecutable. De otra parte están los autores que buscan una “intención” para determinar este carácter. Este grupo de autores lleva incluso a la conclusión que hay clases de tratados que, en atención a su materia, pueden presumirse como autoejecutables. Tal es el caso de los tratados internacionales de derechos humanos. En cualquier caso, ambas posiciones tiene en común la irrelevancia de la distinción entre validez y aplicabilidad de acuerdo al derecho interno y de la doble naturaleza del tratado como norma internacional y como norma interna.

La doctrina nacional mayoritaria adopta explícitamente ${ }^{32}$ el concepto propuesto por JiMÉNEZ DE ARECHAGA de normas autoejecutables: "La estipulación de un tratado se considera como ejecutable por sí misma (self-executing) cuando es susceptible de una aplicación inmediata y directa sin que sea necesaria una acción jurídica complementaria para su implementación o su exigibilidad"33. Así, habría consenso en la doctrina sobre la implicancia del carácter autoejecutable de una disposición de un tratado internacional.

Sin embargo, para un análisis más preciso de la doctrina nacional, es necesario distinguir entre el carácter autoejecutable o no autoejecutable de una disposición (que es donde hay consenso), de las condiciones que determinan ese carácter. Es en este segundo aspecto en donde hay diferencias en la doctrina nacional. Algunos le dan mayor importancia a las condiciones denominadas objetivas (la precisión normativa), mientras que otros prefieren desprender el carácter autoejecutivo o no autoejecutivo de supuestos subjetivos (la intención de las partes).

\footnotetext{
32 Por todos ver Irigoin Barrenne, Jeannette (1995). "Auto-ejecutividad de las disposiciones de la Convención Americana de Derechos Humanos”. En: Solución judicial de controversias: El Derecho Internacional ante los tribunales internacionales e internos, Santiago, Instituto de Estudios Internacionales Universidad de Chile, pp. 33-34.

33 Jiménez de AréCHAGA, Eduardo (1988). "La convención interamericana de derechos humanos como derecho interno". Revista del Instituto Interamericano de DDHH, o 7, p. 26. Así, por ejemplo, Humberto Nogueira propone de manera casi idéntica, "El carácter de autoejecutividad de las normas de un tratado consiste en la posibilidad de aplicar sus disposiciones directamente en el derecho interno sin necesidad de obtener un desarrollo legislativo previo". Nogueira AlCalá, Humberto (1997). "Los Tratados Internacionales en el Ordenamiento Jurídico Chileno". Ius et Praxis, Vol. 2, No 2, pp. 9-62.
} 
Santiago BenADAVA ha afirmado que "[p]ara que las cláusulas de un tratado internacional puedan ser aplicadas por los tribunales internos es necesario que dichas cláusulas sea self-executing, es decir, que tengan precisión normativa suficiente para ser aplicadas directamente en los casos que dichos tribunales conozcan". En cambio -insiste este autor- "si las cláusulas carecen de esta precisión normativa, si no son suficientemente completas y detalladas, ellas requieren, para ser aplicadas internamente, que se dicten medidas legislativas y reglamentarias que las desarrollen" ${ }^{34}$.

La autoejecutabilidad o no autoejecutabilidad se determina en esta posición, por la especificidad de la redacción normativa. En el contexto del debate nacional, se observa que BENADAVA conceptualiza a las disposiciones autoejecutables o no autoejecutables desde una perspectiva objetiva ${ }^{35-36}$.

Humberto Nogueira, en cambio, establece que la condición determinante es la intención de las partes. Así, "estamos en presencia de normas autoejecutables por sí mismas en un tratado, cuando la intención de las partes ha sido garantizar un derecho exigible a las personas que soliciten a los organismos del estado la aplicación de esa norma a su favor" ${ }^{\prime 3}$. Esta es una formulación paradigmática de una perspectiva subjetiva.

La discusión comienza en el momento de determinar las condiciones que configuran y determinan cada categoría de las disposiciones normativas o los tratados mismos. Y esta situación es más debatida. En efecto, si ha de considerarse más preponderante la intención de las partes, es posible, como de hecho lo hace NogueIrA $^{38}$, resolver ex ante, o presuponer el carácter autoejecutable o no de las disposiciones de un tratado internacional (y, aun, del tratado mismo). Por el contrario, considerar el nivel de precisión normativa de las disposiciones, como condición fundamental, no posibilita establecer una presunción del carácter autoejecutivo o no autoejecutivo.

\footnotetext{
34 Benadava, Santiago (1992). "Las Relaciones entre Derecho Internacional y Derecho Interno ante los tribunales chilenos". En: Nuevos enfoques del Derecho Internacional, AA.VV., Santiago: Editorial Jurídica de Chile, p. 42.

${ }^{35}$ Roland Bank ha indicado que la doctrina reconocía tanto condiciones objetivas como subjetivas para la determinación del carácter autoejecutables o no de las disposiciones de tratados internacionales. Las primeras se refieren a si las condiciones y consecuencias de la disposición están claramente definidas - "precisión normativa”- y las segundas si fue la intención de las partes establecer disposiciones de aplicación directa. BANK, Roland (2003). "Tratados internacionales de derechos humanos bajo el ordenamiento jurídico alemán”. Ius et Praxis, Vol. 9, No 1, pp. 23-38.

${ }^{36}$ Irigoin Barrenne (1995). El texto de Jiménez de Arechega consultado directamente y al cual la profesora Irigoin hace referencia es JimÉNEZ dE ARÉCHAGA (1988), p. 26.

37 Nogueira Alcalá, Humberto (2000). "Las Constituciones y los Tratados en materia de Derechos Humanos: América Latina y Chile". Ius et Praxis, Vol. 6, No 2, p. 245. Esta cuestión la repite el mismo autor de manera textual en Nogueira Alcalá (1997), p. 37.

${ }^{38}$ Nogueira Alcalá (2000), pp. 245-246.
} 
Los autores nacionales que defienden una perspectiva objetiva para que una norma sea considerada autoejecutable, debe satisfacer las siguientes condiciones: primero, debe ser una norma de la cual sea posible derivar en forma directa un derecho o una pretensión en favor de un individuo que tenga un interés legítimo en la aplicación de la regla en su caso y que comparece ante el juez o el administrador solicitando esa aplicación; en segundo lugar, la regla debe ser lo suficientemente especifica como para poder ser aplicada judicialmente sin que su ejecución esté subordinada a un acto legislativo o a medidas administrativas subsiguientes ${ }^{39}$.

Desde la perspectiva subjetiva, la existencia en un tratado de una disposición autoejecutable depende principalmente de la intención de las partes contratantes. Esta es la condición subjetiva para su aplicación inmediata y directa y respecto de la cual debe asumirse la interpretación del tratado ${ }^{40}$.

Es en tal sentido que la doctrina mayoritaria presupone, por ejemplo, que las disposiciones contenidas en tratados internacionales de derecho humanos son autoejecutables. Nogueira, establece incluso que "el principio de eficacia directa o autoejecutividad de los derechos humanos, está dado por el hecho de que las normas sobre derechos tienen aplicación directa, salvo que sus enunciados normativos llamen a una intervención necesaria del legislador para otorgarles plena vigencia. Este principio busca impedir que se retarde o bloquee la aplicación de ellos mientras se dictan normas reglamentarias o secundarias. Dejar de cumplir los derechos constituye una forma de inconstitucionalidad por omisión, como asimismo dejar de cumplir los derechos asegurados en tratados constituye violar el tratado y la consiguiente responsabilidad estatal por los actos de sus agentes" ${ }^{\prime 1}$.

Nogueira, haciendo una interpretación del régimen de tratados en la Carta Fundamental, sostiene que ella, en su artículo 50 No 1 , fundamenta la validez de los tratados, y no su aplicabilidad, porque establece precisamente el mecanismo de incorporación del tratado al derecho interno. Pero una vez incorporado, sería el propio tratado el que determina por sí mismo las condiciones de su aplicación. Señala Nogueira que "hoy es indiscutible que los tratados de derechos humanos ratificados por Chile y que se encuentren vigentes son parte del derecho chileno y aplicables de inmediato si se trata de derechos civiles y políticos. Esto constituye una obligación para todos los órganos del estado, incluidos los órganos jurisdiccionales a los que les cabe un rol fundamental en la materia" 42.

Jeannette Irigoin, refiriéndose a la Convención Americana de Derechos Humanos, sostiene un punto similar señalando que "la naturaleza de los trata-

\footnotetext{
${ }^{39}$ Esta segunda condición objetiva es presupuesta por Benadava (1992), p. 42. También Nogueira Alcalá (2000). También Irigoin Barrenne (1995).

${ }^{40}$ Esta condición es la que subyace a la conceptualización que efectúa Humberto Nogueira en Chile. Cf. Nogueira Alcalá (1997), p. 37.

41 Ídem.

${ }^{42}$ Nogueira Alcalá (2000), pp. 262-263.
} 
dos relativos a los derechos humanos cuyo objetivo y fin es la protección de los derechos esenciales de las personas mediante la creación de un sistema de orden público internacional (...) hace indispensable que la mayoría de sus disposiciones sean autoejecutables" ${ }^{33-44}$.

El propósito, según se desprende de manera clara de las posiciones de los autores comentados y de la doctrina mayoritaria ${ }^{45}$, es establecer no solo la presunción de autoejecutabilidad de las disposiciones de los tratados en materia de derechos humanos, sino que, además, restringir la consideración de disposiciones no autoejecutables solo por texto expreso o por la amplitud de la redacción de la disposición normativa (el llamado carácter programático). Pero además, es claro que, al mezclar, como lo hace la doctrina mayoritaria, las condiciones (objetivas y subjetivas) para establecer el carácter autoejecutable o no autoejecutable de las disposiciones de los tratados internacionales, la determinación de una disposición como no autoejecutable, queda circunscrita a un ámbito mínimo. Esta es justamente la lectura que la doctrina en comento ha hecho de la jurisprudencia de los tribunales superiores de justicia cada vez que se ha aplicado normas de tratados internacionales sobre derechos humanos. El razonamiento que subyace en la jurisprudencia, aun cuando no utiliza de manera explícita estas categorías, parece mostrar que la jurisprudencia está adoptando de manera paulatina, la convicción por la presunción de autoejecutabilidad de las disposiciones de los tratados internacionales de Derechos Humanos.

El problema de la aplicación directa de los tratados internacionales solo surge, como se ha insistido, en países cuyo Derecho Constitucional tenga una cláusula general, expresa o tácita, de aplicabilidad directa de los tratados internacionales ${ }^{46}$. La Constitución de los Estados Unidos contiene dicha cláusula en su art. VI inc. $2^{\circ}$. Esta exigencia es clara desde la perspectiva de la teoría del derecho. Como se mencionó, la distinción entre validez y aplicabilidad de normas (o si se quiere, entre validez como pertenencia y validez como obligatoriedad) supone asumir la validez de un enunciado sobre una norma que establezca que: "La norma $\mathrm{N}_{\mathrm{i}}$ es aplicable al caso individual C' porque hay otra norma $\mathrm{N}_{\mathrm{J}}$ que pertenece al sistema jurídico $S_{\mathrm{J}}$ en $\mathrm{T}_{0}$ que establece que $\mathrm{N}_{\mathrm{i}}$ debe ser utilizada en $\mathrm{C}^{\prime \prime} 47$.

\footnotetext{
43 Irigoin BarRenne (1995), p. 32. El resaltado es mío.

${ }^{44}$ La posición crítica en la doctrina nacional es representada por, Correa GonZÁlEz, Rodrigo (2004). “Derogación tácita de normas legislativas por normas de tratados internacionales”. Revista de Derecho UAI. También, del mismo, Correa (2005) y Fuentes Torrijo (2007).

45 Que es, según hemos visto, homogénea en el tratamiento del concepto de autoejecutabilidad y no autoejecutabilidad de disposiciones de tratados internacionales de derechos humanos.

${ }^{46}$ Explícitamente, Correa González (2004), p. 538. En un sentido similar, también, Montt Oyarzún, Santiago (2005). "Aplicación de los tratados bilaterales de protección de inversiones por tribunales chilenos". Revista Chilena de Derecho, Vol. 32, No 1, pp. 19-78.

${ }^{47}$ La formulación, con variantes, corresponde a MORESO (1997), p. 153.
} 
Como se ha mencionado, el caso chileno es distinto. No hay ninguna cláusula expresa que establezca la aplicabilidad directa de los tratados internacionales. Aquí está el problema que configura el debate nacional por la autoejecutabilidad de los tratados internacionales. La adopción de un sistema de ejecución interna general de normas de tratados internacionales ha sido obra de la doctrina y la jurisprudencia nacional en ausencia de un texto expreso constitucional o legal ${ }^{48}$. Tampoco es correcto sostener que el carácter de la autoejecutabilidad puede prevenir de la interpretación de la voluntad de las partes en hacerlo autoejecutable. Esta incorrección proviene nuevamente de la imposibilidad de la doctrina de distinguir entre la naturaleza de norma internacional del tratado y su naturaleza de derecho interno.

En resumen, desde la perspectiva chilena, aunque las categorías son reconocidas por la doctrina desde hace un tiempo y la jurisprudencia del TC las incorporó de manera expresa, no parece claro que la discusión en torno a ellas haya podido clarificar las implicancias jurídicas. Al contrario, una revisión somera de la doctrina ha demostrado que sobre estas categorías la discusión ha sido superficial sin ninguna consideración de la metodología del Derecho Comparado. En efecto, si el presupuesto básico de esta metodología sugiere que sea el principio de funcionalidad el criterio de comparación, en la discusión nacional se ha prescindido absolutamente de esta consideración.

\section{A MODO DE CONCLUSIÓN: APORTACIONES PARA UN DEBATE INCONCLUSO}

El panorama expuesto sobre la consideración y el tratamiento dogmático por parte de la doctrina nacional de las categorías comentadas demuestra el déficit en el tratamiento, sin considerar una perspectiva de Derecho Comparado. Por el contrario, lo que se ha visto es que cada autor e, incluso, el mismo TC, han utilizado estas categorías para los usos más diversos sin un estándar común.

La presunción de autoejecutabilidad es, por lo tanto, equivocada en los términos que se plantean desde la perspectiva del Derecho Comparado. Esta se basa en una mala comprensión de los criterios procedimentales de validez y de los criterios de aplicabilidad del Derecho. En el caso norteamericano su tratamiento obedece a características propias del derecho y de la historia institucional. La doctrina nacional sólo ha reproducido la confusión.

Una tesis asentada en el Derecho Comparado ${ }^{49}$ es que, independientemente del convencimiento en el cumplimiento de las obligaciones internacionales, solo una interpretación fiel del derecho "doméstico" (constitucional) de cada país es lo que posibilita el compromiso de las cortes con el reconocimiento de los derechos consagrados en los tratados internacionales. Esto bajo el supuesto que este trabajo

\footnotetext{
${ }^{48}$ Montt Oyarzún (2005), p. 28.

49 SLoss, David (2009). The Role of Domestic Courts in Treaty Enforcement. A comparative study. Cambridge: Cambridge University Press (2009), p. 210.
} 
quiere dejar planteado que la mejor forma de cumplir con las obligaciones internacionales es el cumplimiento directo en las cortes.

Como señalan ZweigerT y KöTz ${ }^{50}$, la perspectiva del Derecho Comparado es ante todo una perspectiva de clarificación o purificación en el entendido de plantear una perspectiva novedosa que provea de criterios para un análisis en profundidad de las propias instituciones.

Una conclusión que es posible desprender de lo dicho es que, en contextos de un estado de Derecho Constitucional, la presunción de autoejecutabilidad es una tesis equivocada. El error radica en asumir una regla de reconocimiento que no se condice con los criterios procedimentales de validez contemplados en el Derecho Constitucional chileno. Esta regla ha sido deducida más bien del procedimiento que contempla la Constitución para la incorporación de los tratados internacionales al derecho nacional. La solución del problema de la autoejecutabilidad es una cuestión de derecho doméstico. Que se plantea solo una vez que el tratado internacional ha sido incorporado al derecho interno como norma válida. La determinación anterior a este momento supone desconsiderar tanto la naturaleza dual de las normas internacionales como la distinción de validez y aplicabilidad de las normas.

\section{BiBLIOGRAFÍA CITADA}

Alchourrón, Carlos y Bulygin, Eugenio (1974). Introducción a la metodología de las ciencias sociales y jurídicas. Buenos Aires: Astrea.

Alchourrón, Carlos y Bulygin, Eugenio (1991). Análisis lógico y derecho. Madrid: Centro de Estudios Constitucionales.

BANK, Roland (2003). "Tratados internacionales de derechos humanos bajo el ordenamiento jurídico alemán”. Ius et Praxis, Vol. 9, No 1, pp. 23-38.

Bederman, David J. (2008). "Medellin's new paradigm for treaty interpretation”. American Journal of International Law.

Benadava, Santiago (1992). "Las Relaciones entre Derecho Internacional y Derecho Interno ante los tribunales chilenos". En: Nuevos enfoques del Derecho Internacional, AA.VV., Santiago: Editorial Jurídica de Chile.

BRADLEY, Curtis (2008). "Medellin: Intent, presumptions and non self-executing treaties". American Journal of International Law.

Bradley, Curtis (2009). "Self-execution and treaty duality". Supreme Court Review, pp. 131-182.

Bustos Valderrama, Crisólogo (1997). "La incorporación de los tratados en el derecho interno chileno. Análisis desde la perspectiva del Derecho Internacional". Ius et Praxis, Vol. 2, No 2, pp. 73-80.

50 ZweigerT y Kötz (1996), pp. 17 y 18. 
Cena Egaña, José Luis (1997). "Los Tratados de Derechos Humanos y la Constitución Política de la República". Ius et Praxis, Vol. 2, No 2, pp. 81-92.

Correa GonzÁlez, Rodrigo (2000). "Los tratados Internacionales bajo el embrujo de Brown". En: SELA 2000, Seminario en Latinoamérica de Teoría Constitucional y Política.

Correa GonzÁlez, Rodrigo (2004). "Derogación tácita de normas legislativas por normas de tratados internacionales". Revista de Derecho UAI.

Flaherty, Martin S. (1999). "History Right? Historical Scholarship, Original Understanding, and Treaties as 'Supreme Law of the Land'. Columbia Law Review, Vol. 99, p. 2095.

Fuentes Torrijo, Ximena (2007). "El derecho internacional y el derecho interno: Definitivamente una pareja dispareja”. En: SELA 2007, Seminario en Latinoamérica de Teoría Constitucional y Política. Disponible en: <http://www.law. yale.edu/documents/pdf/sela/XimenaFuentes_Spanish_.pdf >.

INFANTE CAFFi, María Teresa (1999). "Los Tratados en el derecho interno chileno: El efecto de la reforma constitucional de 1989 visto por la jurisprudencia”. Revista Chilena de Derecho, Vol. 23, No 2 y 3, pp. 277-297.

Irigoin Barrenne, Jeannette (1995). "Auto-ejecutividad de las disposiciones de la Convención Americana de Derechos Humanos”. En: Solución judicial de controversias: El Derecho Internacional ante los tribunales internacionales e internos, Santiago, Instituto de Estudios Internacionales Universidad de Chile.

IWASAWA, Yuji (1986). "The doctrine of self-executing treatise in the United States: A critical analysis". Virginia Journal of Internacional Law, No 26, pp. 627-692.

JimÉNEZ DE ARÉCHAGA, Eduardo (1988). "La convención interamericana de derechos humanos como derecho interno". Revista del Instituto Interamericano de DDHH, No 7, pp. 25-41.

Montt Oyarzún, Santiago (2005). "Aplicación de los tratados bilaterales de protección de inversiones por tribunales chilenos". Revista Chilena de Derecho, Vol. 32, No 1, pp. 19-78.

Moreso, Josep Joan (1997). La indeterminación del Derecho y la interpretación de la Constitución. Madrid: Centro de Estudios Constitucionales.

Morison, Samuel Eliot; Commager, Henry Steele y Leuchtenburg, William E. (2013). Breve Historia de los Estados Unidos de América. México D.F.: Fondo de Cultura Económica.

Nogueira Alcalá, Humberto (1997). "Los Tratados Internacionales en el Ordenamiento Jurídico Chileno". Ius et Praxis, Vol. 2, No 2, pp. 9-62.

Nogueira Alcalá, Humberto (2000). "Las Constituciones y los Tratados en materia de Derechos Humanos: América Latina y Chile”. Ius et Praxis, Vol. 6, No 2, pp. 227-279. 
Pfeffer Urquiaga, Emilio (1997). "Constitución Política de la República y Tratados Internacionales". Ius et Praxis, Vol. 2, No 2, pp. 63-72.

Schmidt Hotт, Claudia (1999). "Filiación matrimonial y filiación no matrimonial: Fundamento y alcance de esta distinción en la nueva legislación”. En: El nuevo estatuto de fliación en el Código Civil chileno, Santiago: Fundación Facultad de Derecho.

SLOSS, David (2002). "Non self-executing Treatise: Exposing a Constitutional Fallacy". U.C. Davies Law Review, Vol. 36, No 1, pp. 3-84.

SLoss, David (2009). The Role of Domestic Courts in Treaty Enforcement. A comparative study. Cambridge: Cambridge University Press.

Sloss, David (2012). "Executing Foster v. Neilson: The Two-Step Approach to Analyzing Self-Executing Treaties". Harvard International Law Journal, Vol. 53, pp. 302-354.

VÁzQuez, Carlos Manuel (1995). “The four doctrines of self-executing treaties”. The American Journal of International Law, Vol 89, No 4, pp. 695-723.

VÁzQuez, Carlos Manuel (1999). "Laughing at Treaties". Columbia Law Review, Vol. 99, pp. 2154-2217.

VÁzQueZ, Carlos Manuel (2008). "Treaties as Law of the Land: The Supremacy Clause and the Judicial Enforcement of Treaties". Harvard Law Review, Vol. 122, pp. 599-695.

Von Wrigth, George Henrik (1963). Norm and Action. A Logical Enquiry. London: Routledge \& Kegan Paul.

Yoo, John (1999). "Treaties and Public Lawmaking: A Textual and Structural Defense of Non-Self-Execution”. Columbia Law Review, Vol. 99, pp. 22182258.

Zweigert, Konrad and KöTz, Hein (1996). Introduction to Comparative Law. Oxford: Clarendon Press, Third Edition.

\section{JURISPRUDENCIA COMPARADA CITADA}

Foster \& Elam v. Neilson, 27 U.S. 2 Pet. 253253 (1829).

Ware v. Hylton, 3 U.S. 3 Dall. 199199 (1796).

United States v. Percheman, 32 U.S. 7 Pet. 5151 (1832).

Medellin v. Texas 552 U.S. 491 (2008). 